\title{
Two dimensional blue native / blue native polyacrylamide gel electrophoresis for the characterization of mitochondrial protein complexes and supercomplexes
}

Stephanie Sunderhaus, Holger Eubel and Hans-Peter Braun

Prof. Dr. Hans-Peter Braun

Abteilung Angewandte Genetik

Naturwissenschaftliche Fakultät

Universität Hannover

Herrenhäuser Str. 2

30419 Hannover, Germany

Tel: $\quad+495117622674$

Fax: $\quad+495117623608$

braun@genetik.uni-hannover.de

Running head: 2D blue native / blue native PAGE

Key words: $\quad$ Blue-native polyacrylamide gel electrophoresis (BN-PAGE), digitonin, in gel enzyme assay, membrane proteins, mitochondria, protein complexes, protein supercomplexes, respiratory chain 


\begin{abstract}
Blue-native polyacrylamide gel electrophoresis (BN PAGE) employs the blue wool dye Coomassie for labeling of proteins and protein complexes under native conditions. Electrophoresis under native conditions subsequently allows resolving proteins and protein complexes according to their molecular mass. BN-PAGE can be combined with second gel dimensions for extended analyses. Best known is the 2D BN / SDS PAGE system, which allows resolution of subunits of protein complexes. Recently, a 2D BN / BN PAGE system was suggested, which proved to be useful for investigating the substructure of protein complexes and protein supercomplexes. Basis of this 2D system is a variation in the conditions used for the two BN gel dimensions. Here we present a basic protocol for the analysis of mitochondrial fractions by 2D BN / BN PAGE. The 2D BN / BN system is compatible with in gel enzyme activity stainings, because both gel dimensions are carried out under native conditions.
\end{abstract}




\section{Introduction}

Blue-native polyacrylamide gel electrophoresis (BN-PAGE) is based on incubation of proteins with Coomassie-blue. Coomassie belongs to the trimethylmethane dye family and was used as a wool dye since the late $19^{\text {th }}$ century. Due to its efficient and specific binding to proteins it was introduced in biochemistry to visualize proteins on gels after electrophoresis and later to photometrically determine protein concentrations [1,2]. Coomassie is a negativelycharged compound which carefully introduces charges into proteins and protein complexes. Because of this property it was suggested to be used for protein labeling prior to electrophoretic separations [3]. Coomassie does not denature proteins. In combination with non-ionic detergents, BN-PAGE proved to be an ideal tool for the analysis of membrane bound protein complexes, especially for those of mitochondria [3]. Even supramolecular assemblies of membrane bound mitochondrial protein complexes were shown to be stable during BNPAGE, if the membrane solubilization step is carried out under very mild conditions [4]. Classically, a first Blue-native gel dimension is combined with SDS-PAGE as a second gel dimension, which allows to separate subunits of protein complexes. On the resulting gels, subunits of protein complexes form vertical rows. Detailed protocols for 2D BN / SDS PAGE were published recently [5-7].

Five years ago, a novel two-dimensional gel electrophoresis system was suggested which is based on BN-PAGE for both gel dimensions [4]. If the two gel dimensions are carried out under identical conditions, protein complexes form a diagonal line on the resulting 2D gels. However, if conditions of the second dimension BN-PAGE are slightly less gentle than conditions of the first dimension BN-PAGE, protein complexes are dissected into subcomplexes, which are visible underneath this diagonal line. 2D BN / BN PAGE proved to be a very powerful tool for the investigation of the protein complex composition of supercomplexes or the subcomplex composition of protein complexes. It successfully was used to characterize the supramolecular structure of the protein complexes of the oxidative phosphorylation (OXPHOS) system in mitochondria [4, 6, 8-14] and of the photosynthetic electron transport system in chloroplasts [15]. Conditions to be varied between the two BN gel dimensions can refer to detergent type (e.g. digitonin / dodecylmaltoside), detergent concentration (e.g. 0.5 / 2.0 $\%$ dodecylmaltoside), temperature (e.g. $4^{\circ} / 20^{\circ} \mathrm{C}$ ) or presence of chaotropic compounds (absence / presence of urea). 2D BN / BN PAGE is compatible with in gel enzyme stainings, allowing to determine the activity of supramolecular assemblies of protein complexes as well 
as the activities of subcomplexes of protein complexes (Fig. 1). In this chapter, we present a basic protocol for the analysis of mitochondrial protein complexes by BN / BN PAGE, which is based on detergent variation between the two $\mathrm{BN}$ gel dimensions. The presented protocol also is suitable for the analysis of other organell fractions or bacteria.

\section{Materials}

\subsection{Preparation of $B N$ gels for first and second gel dimensions}

1. Acrylamide solution: $49.5 \%$, acryl / bisacryl = 32 / 1 (AppliChem, Darmstadt, Germany)

2. Gel buffer BN (6x): 1.5 M amino caproic acid, $150 \mathrm{mM}$ BisTris, $\mathrm{pH} 7.0$ (adjust at $\left.4^{\circ} \mathrm{C}\right)$

\subsection{Sample preparation}

1. Digitonin solubilization solution: $5.0 \%$ digitonin (e.g. Fluka, Buchs, Switzerland), $30 \mathrm{mM}$ HEPES, $150 \mathrm{mM}$ potassium acetate, $10 \%$ (v/v) glycerol, $\mathrm{pH} 7.4$ (adjust at $4^{\circ} \mathrm{C}$ ). This buffer should be freshly prepared and shortly heated to $98^{\circ} \mathrm{C}$ to dissolve the detergent. Add PMSF directly before use (final concentration: $2 \mathrm{mM}$; stock solution: $200 \mathrm{mM}$ PMSF [w/v] in EtOH)

2. Coomassie-blue solution: 5 \% Coomassie G 250 (e.g. Merck, Darmstadt, Germany), $750 \mathrm{mM}$ amino caproic acid

\subsection{First dimension BN-PAGE}

1. Cathode buffer BN (5x): $250 \mathrm{mM}$ Tricine, $75 \mathrm{mM}$ BisTris, $0.1 \%(\mathrm{w} / \mathrm{v})$ Coomassie G 250 (e.g. Merck, Darmstadt, Germany), pH 7.0 (adust at $4^{\circ} \mathrm{C}$ )

2. Anode buffer $\mathrm{BN}(6 \mathrm{x}): 300 \mathrm{mM}$ BisTris, $\mathrm{pH} 7.0$ (adjust at $4^{\circ} \mathrm{C}$ )

\subsection{Transfer of gel stripes of first gel dimensions onto second gel dimensions}

1. 1x Cathode buffer $\mathrm{BN}+$ dodecylmaltoside: $50 \mathrm{mM}$ Tricine, $15 \mathrm{mM}$ BisTris, $0.03 \%$ dodecylmaltoside, 0.02 (w/v) Coomassie G 250 (e.g. Merck, Darmstadt, Germany), $\mathrm{pH} 7.0$ (adjust at $4^{\circ} \mathrm{C}$ ) 


\subsection{Second dimension BN-PAGE}

1. Agarose solution: $1.5 \%(\mathrm{w} / \mathrm{v})$ Agarose

2. Cathode buffer BN + dodecylmaltoside: (see 2.4.)

3. Anode buffer BN (6x): (see 2.3.)

\subsection{Enzyme activity staining procedures of 2D BN / BN gels}

1. Phosphate buffer stock solution: $1 \mathrm{M}$ phosphate, $\mathrm{pH} 7.4$

2. DAB stock solution: 0.1 M 3,3'-diamino benzidine tetrahydrochloride dihydrate

3. KCN stock solution: $1 \mathrm{M} \mathrm{KCN}$

4. EDTA stock solution: 0.1 M EDTA

5. Tris stock solution: $2 \mathrm{M}$ Tris- $\mathrm{HCl}, \mathrm{pH} 7.4$

6. Cytochrome c oxidase staining solution: $10 \mathrm{mM}$ Phosphate buffer, $\mathrm{pH} 7.4,0.1 \%$ (w/v) DAB, $7.5 \%(\mathrm{w} / \mathrm{v})$ sucrose, $19 \mathrm{U} / \mathrm{ml}$ catalase and $16 \mathrm{mM}$ cytochrome c (Sigma-Aldrich, St. Louis, MO, USA)

7. Succinate dehydrogenase staining solution: $50 \mathrm{mM}$ Phosphate buffer, $\mathrm{pH}$ 7.4, 84 $\mathrm{mM}$ succinic acid, $0.2 \mathrm{mM}$ N-Methylphenazonium methyl sulfate (Fluka, Buchs, Switzerland), $0.2 \%$ Nitro tetrazolium blue (Fluka, Buchs, Switzerland), $4.5 \mathrm{mM}$ EDTA, $10 \mathrm{mM} \mathrm{KCN}$

8. NADH dehydrogenase staining solution: 0.1 M Tris-HCl, $\mathrm{pH} 7.4,0.14 \mathrm{mM}$ NADH, $0.1 \%$ Nitro tetrazolium blue (Fluka, Buchs, Switzerland)

9. Fixing solution: $40 \%(\mathrm{v} / \mathrm{v})$ methanol, $10 \%(\mathrm{w} / \mathrm{v})$ acetic acid

\section{Methods}

Digitonin currently is considered to be the mildest detergent for mitochondrial research, which better stabilizes most supramolecular structures than Triton X100, dodecylmaltoside or other non-ionic detergents. In the protocol given below, first dimension BN-PAGE is therefore carried out after membrane solubilization using digitonin and second dimension $\mathrm{BN}$ PAGE after incubation of the gel stripe of the first gel dimension with dodecylmaltoside. However, numerous variations of this experimental design are possible (see Note 1) 


\subsection{Preparation of BN gels for first and second gel dimensions}

Best resolution capacity of $\mathrm{BN}$ gels is achieved if the electrophoretic separation distance is > $12 \mathrm{~cm}$. The following instructions refer to the Protean II electrophoresis unit (BioRad, Richmond, Ca, USA; dimensions $0.1 \times 16 \times 20 \mathrm{~cm}$ for the gel of the first dimension, $0.15 \times 16 \times$ $20 \mathrm{~cm}$ for the gel of the second dimension). However, units from other manufacturers are of comparable suitability for BN-PAGE, e.g. the Hoefer SE-400 or SE-600 gel systems (Amersham Biosciences, Uppsala, Sweden). Usage of gradient gels is recommended, because molecular masses of protein complexes can vary between $50 \mathrm{kDa}$ and several thousand $\mathrm{kDa}$. (see Note 2)

First dimension gel (thickness of the gel: $1.0 \mathrm{~mm}$ )

1. Prepare a $4.5 \%$ separation gel solution by mixing $1.2 \mathrm{ml}$ Acrylamide solution with $2.2 \mathrm{ml}$

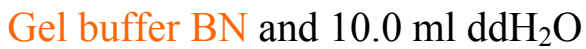

2. Prepare a $16 \%$ separation gel solution by mixing $4.5 \mathrm{ml}$ Acrylamide solution with $2.2 \mathrm{ml}$

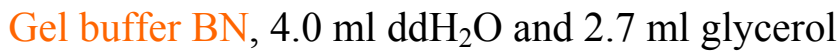

3. Transfer the two gel solutions into the two chambers of a gradient former and connect the gradient former via a hose and a needle with the space in-between two glass plates, which are pre-assembled in a gel casting stand. Gradient gels can either be pored from the top (16\% gel solution has to enter the gel sandwich first) or from the bottom ( $4.5 \%$ gel solution has to enter first). Poring gradient gels at $4^{\circ} \mathrm{C}$ is recommended to avoid premature polymerization.

4. Add APS and TEMED to the two gel solutions (60 $\mu 110 \%$ APS / $6 \mu 1$ TEMED to the 4.5 $\%$ gel solution, $40 \mu \mathrm{l}$ APS / $4 \mu 1$ TEMED to the $16 \%$ gel solution)

5. Pour the gradient gel, leaving space for the stacking gel, and overlay with $\mathrm{ddH}_{2} \mathrm{O}$. The gel should polymerize in about 60 minutes

6. Pour off the $\mathrm{ddH}_{2} \mathrm{O}$

7. Prepare the stacking gel solution by mixing $1.2 \mathrm{ml}$ Acrylamide solution, $2.5 \mathrm{ml}$ Gel buffer

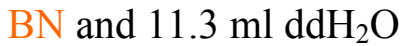

8. Add $65 \mu$ APS and $6.5 \mu 1$ TEMED and pour the stacking gel around an inserted the comb. The stacking gel should polymerize within 30 minutes. 
Second dimension gel (thickness of the gel: $1.5 \mathrm{~mm}$ )

1. Prepare a $5.0 \%$ separation gel solution by mixing $2.0 \mathrm{ml}$ Acrylamide solution with $3.3 \mathrm{ml}$ Gel buffer $\mathrm{BN}$ and $14.7 \mathrm{ml} \mathrm{ddH_{2 } \mathrm { O }}$

2. Prepare a $20 \%$ separation gel solution by mixing $8.0 \mathrm{ml}$ Acrylamide solution with $3.3 \mathrm{ml}$

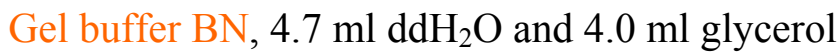

3. Transfer the two gel solutions into the two chambers of a gradient former as described for the first dimension gel

4. Add APS and TEMED to the two gel solutions ( $90 \mu 110 \%$ APS / $9 \mu 1$ TEMED to the $5 \%$ gel solution, $50 \mu$ APS / $5 \mu 1$ TEMED to the $20 \%$ gel solution)

5. Pour the gradient gel, leaving space for the stacking gel, and overlay with $\mathrm{ddH}_{2} \mathrm{O}$. The gel should polymerize in about 60 minutes

6. Pour off the dd $\mathrm{H}_{2} \mathrm{O}$

7. Prepare the stacking gel solution by mixing $1.2 \mathrm{ml}$ Acrylamide solution, $2.5 \mathrm{ml} \mathrm{Gel} \mathrm{buffer}$

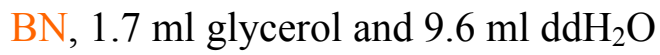

8. Add $65 \mu \mathrm{l}$ APS and $6.5 \mu \mathrm{l}$ TEMED and cast the stacking gel until $1 \mathrm{~cm}$ below the upper edge of the inner glass plate (do not insert a comb). Overlay with $\mathrm{ddH}_{2} \mathrm{O}$. The stacking gel should polymerize within 30 minutes. Finally remove the overlaying water.

Gels for both gel dimensions should be prepared one day before usage and should be stored at $4^{\circ} \mathrm{C}$.

\subsection{Sample preparation}

All steps of the sample preparation should be carried out at $4{ }^{\circ} \mathrm{C}$.

1. Prepare mitochondria of interest (see Note 3)

2. Determine the protein concentration, e.g. according to Lowry [16].

3. Adjust the protein concentration to $10 \mu \mathrm{g} / \mu \mathrm{l}$

4. Centrifuge $50 \mu \mathrm{l}$ fractions (about $0.5 \mathrm{mg}$ protein) for 10 minutes at $15000 \mathrm{xg}$ to sediment organelles

5. Resuspended pellets in $50 \mu 1$ Digitonin solubilization solution

6. Incubate the fractions for 20 minutes on ice 
7. Centrifuge the fractions for 20 minutes at $20000 \mathrm{xg}$ to remove insoluble material

8. Supplement the fractions with $2.5 \mu 1$ Coomassie-blue solution

9. Load $30-50 \mu \mathrm{l}$ of the supernatants (corresponding to about $0.3-0.5 \mathrm{mg}$ mitochondrial protein) directly into the wells of a $\mathrm{BN}$ gel (protein amounts are adjusted to allow staining of gels by Coomassie; if silver staining shall be applied, protein amounts can be reduced by factor 10$)$.

\subsection{First dimension BN-PAGE}

1. Prepare $1 \mathrm{xAnode}$ and $1 \mathrm{xCathode}$ buffers $\mathrm{BN}$ by diluting the corresponding stock solutions

2. Carefully remove the comb of the first dimension BN gel

3. Assemble the gel electrophoresis unit, add 1xCathode and 1xAnode buffers BN to the upper and lower chambers of the gel unit. Cool down the unit to $4^{\circ} \mathrm{C}$

4. Load Coomassie-blue pre-treated protein samples (see 3.2.) into the gel wells

5. Connect the gel unit to a power supply. Start electrophoresis at constant voltage (100V for 45 minutes) and continue at constant current (15 mA for about 5 hours, see Note 4). Electrophoresis should be carried out at $4^{\circ} \mathrm{C}$. Blue gel bands should already be visible during the electrophoresis run.

\subsection{Transfer of gel stripes of first gel dimensions onto second gel dimensions}

1. Cut out a lane of the first dimension BN gel

2. Incubate the gel stripe for 10 minutes in Cathode buffer $\mathrm{BN}+$ dodecylmaltoside at $4{ }^{\circ} \mathrm{C}$

\subsection{Second dimension BN-PAGE}

1. Assemble the gel electrophoresis unit and transfer the gel stripe of the first gel dimension onto a second dimension gel. Make sure it is placed centrally and that is has close contact to the second dimension gel. Fix the gel stripe with $1.5 \%$ agarose solution (boil the solution before use and allow it to cool down to approximately $45^{\circ} \mathrm{C}$ ). (see Note 5 )

2. Prepare $1 \mathrm{xAnode}$ buffer BN by diluting the corresponding stock solution

3. Add 1x Cathode buffer BN + dodecylmaltoside and 1xAnode buffer BN to the upper and lower chambers of the gel unit. 
4. Connect the gel unit to a power supply. Start electrophoresis at constant voltage (100V for 45 minutes) and continue at constant current (15 mA for 6 - 12 hours, see Note 6). Electrophoresis should be carried out at $4^{\circ} \mathrm{C}$. Blue gel spots should already be visible during the electrophoresis run.

\subsection{Enzyme activity staining procedures of 2D BN / BN gels}

After completion of the electrophoresis run, 2D BN / BN gels can be stained using Coomasssie colloidal [17, 18] or silver [19] (see Notes 7 and 8). However, since both gel dimensions are carried out under native conditions, 2D BN / BN PAGE also is compatible with in gel enzyme activity stainings [20,21]. Three classical in gel staining procedures for enzymes of the respiratory chain are given below (activity staining procedures for several other enzymes can be found in the literature).

1. incubate the gel with $\mathrm{ddH}_{2} \mathrm{O}$ for two times 10 minutes

2. incubate the gel with $100 \mathrm{ml}$ freshly prepared staining solution (Cytochrome c oxidase staining solution, or Succinate dehydrogenase staing solution, or NADH dehydrogenase staining solution) Staining takes minutes until hours depending on the abundance of the stained enzyme.

3. Stop the reaction by transferring the gel into Fixing solution (see Notes 9 and 10).

\section{Notes}

1. Conditions to be varied in the two gel dimensions of BN / BN PAGE can refer to detergent type, detergent concentration, temperature, presence of chaotropic compounds and others. Conditions to monitor dissection of supercomplexes into protein complexes and / or dissection of protein complexes into subcomplexes should be optimized for the mitochondria of interest.

2. If very large protein complexes ( $>3 \mathrm{MDa}$ ) have to be resolved, the acrylamide gradient gel of the $\mathrm{BN}$ gel dimension can be substituted by a $2.5 \%$ agarose gel prepared in Gel buffer $\mathrm{BN}[22]$. 
3. Alternatively, mitochondrial subfractions can be used as starting material for $\mathrm{BN} / \mathrm{BN}$ PAGE, e.g. an inner membrane or a matrix fraction.

4. Electrophoresis should not be carried out for more than 5 hours, because protein complexes might get stuck in the pores of the gradient polyacrylamide gel, which might prevent migration of the complexes into the second gel dimension. In general, 2/3 completion of the electrophoresis run is sufficient for protein complex resolution on the first gel dimension.

5. Transfer of a lane of a BN gel onto a second gel dimension is proposed to be carried out by fixing a lane of a first dimension $\mathrm{BN}$ gel onto a pre-poured $\mathrm{BN}$ gel for second gel dimension. By using this procedure, time between first and second gel dimension is minimized, which is advantageous for activity stainings of 2D BN / BN gels. However, physical contact between the lane of the first dimension gel and the second dimension gel might be better if the lane of the second gel dimension is embedded into the stacking gel of the second gel dimension (see [5-7] for corresponding protocols). On the other hand, TEMED and APS of the gel solution for the second gel dimension can diffuse into the gel stripe of the first gel dimension, which usually greatly reduces enzymatic activities. Therefore, this procedure should only be applied for 2D BN / BN gels in combination with Coomassie and silver staining.

6. Electrophoresis of the second gel dimension should be carried out for 6-12 hours or longer. Often, sharpness of protein spots is best after long electrophoresis, because protein complexes get stuck into the pores of the gradient gel of the second gel dimension at defined polyacrylamide concentrations.

7. Protein complexes resolved on $2 \mathrm{D} \mathrm{BN} / \mathrm{BN}$ gels also can be blotted onto membranes. Short pre-blots should be carried out to electrophoretically de-stain gels from excess of Coomassie-blue [23]. Alternatively, the Cathode-buffer BN can be replaced by a Cathode buffer BN without Coomassie blue after $50 \%$ completion of the electrophoresis run of the second BN gel dimension. Furthermore, protein complexes excised from 2D BN / BN gels also can be separated on a third gel dimension, which is carried out in the presence of SDS [4].

8. Protein complexes resolved by 2D BN / BN PAGE also can be cut out and prepared for analysis by mass spectrometry. Usually the subunits of protein complexes are first fragmented by Trypsin. Afterwards, peptides are best analyzed by coupled liquid choromatography and electrospray tamdem mass spectrometry (LC-MS/MS). 
9. Alternatively, in gel activity staining also can be stopped by adding inhibitors of the monitored enzymes

10. If $2 \mathrm{D} \mathrm{BN} / \mathrm{BN}$ gels shall be Coomassie or silver stained after activity staining, wash the gels several times with $\mathrm{ddH}_{2} \mathrm{O}$ before fixation to remove proteins of the activity staining solutions. This will reduce background staining of the gels.

\section{Acknowledgments:}

This work was supported by the Deutsche Forschungsgemeinschaft (grant Br 1829 - 7/1)

\section{References}

[1] Groth, S.F., Webster, R.G. and Datyner, A. (1963) Two new staining procedures for quantitative estimation of proteins on electrophoretic stripes. Biochim. Biophys. Acta 71, 377-391.

[2] Bradford, M. (1976) A rapid and sensitive method for the quantitation of microgram quantities of protein utilizing the principle of protein-dye binding. Anal. Biochem. 72, 248-254.

[3] Schägger, H, and von Jagow, G. (1991) Blue native electrophoresis for isolation of membrane protein complexes in enzymatically active form. Anal. Biochem. 199, 223231.

[4] Schägger, H. and Pfeiffer, K. (2000) Supercomplexes in the respiratory chain of yeasts and mammalian mitochondria. EMBO J. 19, 1777-1783.

[5] Schägger, H. (2001a) Blue-native gels to isolate protein complexes from mitochondria. Meth. Cell Biol. 166, 231-244.

[6] Schägger, H. (2003) Blue native electrophoresis. In: Membrane protein purification and crystallization: A practical guide. C. Hunte, G. von Jagow and H. Schägger (eds), Academic Press, London, UK, pp. 105-130.

[7] Heinemeyer, J., Lewejohann, D. and Braun, H.P. (2005) Blue-native gel electrophoresis for the characterization of protein complexes in plants. In: Methods in Molecular Biology: Plant Proteomics, H. Thiellement, V. Méchin, C. Damerval and M. Zivy (eds), Humana press, in press. 
[8] Schägger H and Pfeiffer K. (2001) The ratio of oxidative phosphorylation complexes $\mathrm{I}-\mathrm{V}$ in bovine heart mitochondria and the composition of respiratory chain supercomplexes.

J. Biol. Chem. 276, 37861-37867.

[9] Schägger, H. (2001b) Respiratory chain super complexes. IUBMB Life 52, 119-128.

[10] Schägger, H. (2002) Respiratory supercomplexes of mitochondria and bacteria. Biochim. Biophys. Acta 1555, 154-159.

[11] Eubel, H., Jänsch, L. and Braun, H.P. (2003) New insights into the respiratory chain of plant mitochondria: supercomplexes and a unique composition of complex II. Plant Physiol. 133, 274-286.

[12] Eubel, H., Heinemeyer, J. and Braun, HP. (2004) Identification and characterization of respirasomes in potato mitochondria. Plant Physiol. 134, 1450-1459.

[13] Millar, A.H., Eubel, H., Jänsch, L., Kruft, V., Heazlewood, L. and Braun, H.P. (2004) Mitochondrial cytochrome c oxidase and succinate dehydrogenase contain plantspecific subunits. Plant Mol. Biol. 56, 77-89.

[14] Krause, F., Reifschneider, N.H., Vocke, D., Seelert, H., Rexroth, S. \& Dencher, N.A. (2004) "Respirasome"-like supercomplexes in green leaf mitochondria of spinach. $J$. Biol. Chem. 279, 48369-48375.

[15] Heinemeyer, J., Eubel, H., Wehmhöner, D., Jänsch, L. and Braun, H.P. (2004) Proteomic approach to characterize the supramolecular organization of photosystems in higher plants. Phytochemistry 65, 1683-1692.

[16] Lowry, O.H., Rosebrough, N.J., Farr, A.L. and Randall, R.J. (1951) Protein measurement with the Folin phenol reagent. J. Biol. Chem. 193, 265-275.

[17] Neuhoff, V., Stamm, R. and Eibl, H. (1985) Clear background and highly sensitive protein staining with Coomassie Blue dyes in polyacrylamide gels: A systematic analysis. Electrophoresis 6, 427-448.

[18] Neuhoff, V., Stamm, R., Pardowitz, I., Arold, N., Ehrhardt, W. and Taube, D. (1990) Essential problems in quantification of proteins following colloidal staining with Coomassie Brilliant Blue dyes in polyacrylamide gels, and their solution. Electrophoresis 11, 101-117.

[19] Heukeshoven, J. \& Dernick, R. (1986) Silver staining of proteins. In: BJ Radola, ed., Elektrophoresis Forum '86, Technische Universität München, p. 22-27.

[20] Grandier-Vazeille, X. and Guerin, M. (1996) Separation by blue native and colorless native polyacrylamide gel electrophoresis of the oxidative phosphorylation complexes 
of yeast mitochondria solubilized by different detergents: specific staining of the different complexes. Anal. Biochem. 242, 248-254.

[21] Zerbetto, E., Vergani, L. and Dabbeni-Sala, F. (1997) Quantitation of muscle mitochondrial oxidative phosphorylation enzymes via histochemical staining of blue native polyacrylamide gels. Electrophoresis 18, 2059-2064.

[22] Henderson, N.S., Nijtmans, L.G., Lindsay, J.G., Lamantea, E., Zeviani, M. and Holt, I.J. (2000) Separation of intact pyruvate dehydrogenase complex using blue native agarose gel electrophoresis. Electrophoresis 21, 2925-2931.

[23] Jänsch, L., Kruft, V., Schmitz, U.K. and Braun, H.P. (1996) New insights into the composition, molecular mass and stoichiometry of the protein complexes of plant mitochondria. Plant J. 9, 357-368.

\section{Figure legend:}

Resolution of mitochondrial protein complexes from Arabidopsis by two-dimensional BN / BN PAGE. Proteins were solubilized by Digitonin solubilization solution. A: 2D BN / BN gel after Coomassie-staining, B: 2D BN / BN gel after activity staining for NADH dehydrogenase (complex I). The numbers to the right refer to the molecular masses of standard proteins. $\mathrm{I}+\mathrm{III}_{2}$ : supercomplex composed of complexes I and $\mathrm{III}_{2}$; I: NADH dehydrogenase (complex I); V: ATP synthase (complex V); III $_{2}$ : dimeric cytochrome c reductase (complex III); IV: cytochrome c oxidase (complex IV), II: succinate dehydrogenase (complex II). Note: activity staining allows to visualize the singular and the supercomplex-bound form of complex I. Furthermore, complex I is partially dissected into fragments of $600 \mathrm{kDa}$ (hydrophobic arm) and $400 \mathrm{kDa}$ (matrix exposed arm). The $400 \mathrm{kDa}$ arm includes the NADH oxidizing domain and therefore is also labeled by activity staining, whereas the $600 \mathrm{kDa}$ arm is not. 
Sunderhaus et al., Figure 1

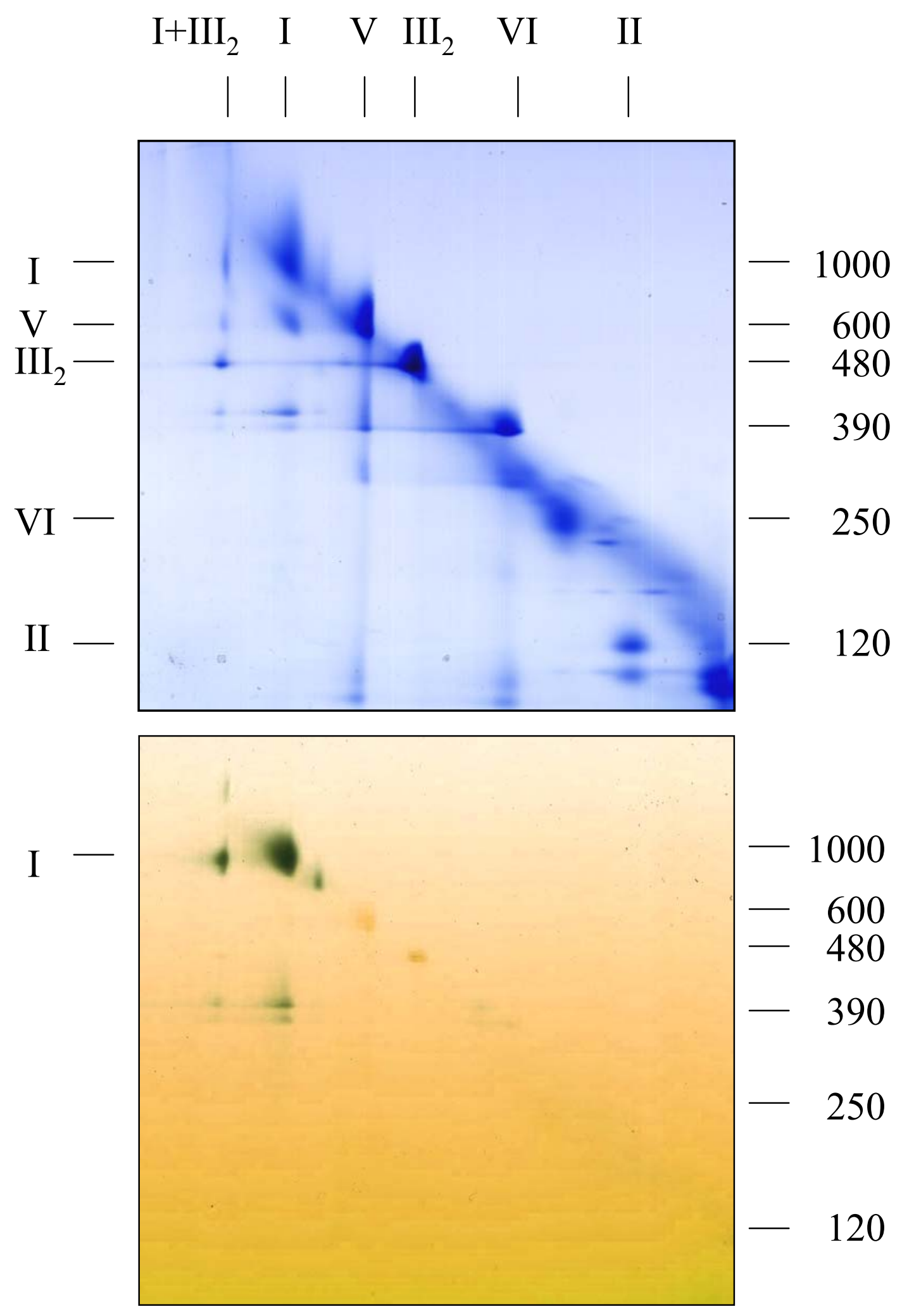

